\title{
Sonhos e craving em alcoolistas na fase de desintoxicação
}

\section{Dreams and Craving in Alcohol Addicted Patients in the Detoxication Stage}

\author{
Renata Brasil Araujo ${ }^{1}$ \\ MARgareth Oliveira ${ }^{2}$ \\ LUCIANE B. PICCOLOTO ${ }^{3}$ \\ KAREN P.D.R SZUPSZYNSKI ${ }^{4}$
}

\begin{abstract}
Resumo
O objetivo desta pesquisa foi avaliar a presença da relação entre os sonhos e craving em alcoolistas nos três primeiros dias de desintoxicação em unidades de internação hospitalar. Verificou-se se aqueles que tinham o craving aumentado relatavam sonhos com o tema “álcool" e analisou-se a qualidade do sono desses sujeitos. Foi um estudo transversal, de associação entre variáveis. A amostra foi de 77 sujeitos adultos do sexo masculino, dependentes de álcool, sem comorbidades clínicas ou psiquiátricas e não-dependentes de outras substâncias psicoativas, salvo a nicotina. Os instrumentos foram: entrevista estruturada; escala de avaliação do craving; questionário de avaliação do sono e dos sonhos; Mini-Mental State Examination e questionário Short-Form Alcohol Dependence Data. Quanto à fase inicial do sono, $67,6 \%$ considerou no mínimo satisfatória, 80,5\% emitiu a mesma opinião quanto ao seu final, porém apenas $22,1 \%$ nunca apresentou interrupções durante o sono. Sonhar com álcool não foi um comportamento freqüente $(27,3 \%)$, e a média de pontuação do craving foi "fraca", havendo associação entre sonhar com álcool e um aumento no craving ( $\mathrm{p}<0,001)$. Os sonhos podem ser mais bem aproveitados pelos profissionais da dependência química, devendo ser utilizados elementos sinalizadores de uma "situação de risco" nas técnicas de prevenção à recaída.
\end{abstract}

Palavras-chave : Sonhos, sono, álcool, craving (fissura). 
have clinical or psychiatric comorbidities. In addition, the subjects would not be addicted to any other psychoactive substance but nicotine. The following instruments have been used: an Information File, where the subjects should fill in their social-demographic status; a Craving Evaluation Questionnaire; a Sleep and Dream Evaluation Questionnaire, Mini-Mental State Examination and Short-Form Alcohol Dependence Data Questionnaire ). $67,6 \%$ of the subjects considered the sleeping initial stage at least "satisfactory", $80,5 \%$ of the subjects stated the same concerning the sleeping final stage, but only $22,1 \%$ of the subjects never presented interruptions while sleeping. Dreaming of "alcohol" had not been a frequent behaviour $(27,3 \%)$ and the average craving rating was "low"; there is a connection between dreaming of alcohol and craving increase $(p<0,001)$. Dreams can be better used by professionals who assist chemical addicted patients, and indicators of a "risk situation" should be used in the techniques of Relapse Prevention.

Keywords : Dreams, sleep, alcohol, craving.

\section{Introdução}

O craving é um fator que pode estar presente tanto durante o uso do álcool, como na fase de desintoxicação, ou ainda, após uma interrupção mais prolongada (Beck et al., 1999), sendo este um dos sinais de alerta que poderia levar a um lapso ou recaída (Hogstrom et al., 1999).

Imagens mentais associadas ao álcool podem interferir no aumento do craving, e este conhecimento auxilia o paciente em termos de auto-eficácia, pois o instrumentaliza a entender que determinadas situações ou cenas devem ser evitadas para não comprometer sua abstinência (Weinstein et al., 1998).

À medida que $100 \%$ dos sonhos são compostos de imagens visuais (Reimão, 1996), o material onírico pode ser utilizado como mais um instrumento para avaliar o craving em indivíduos alcoolistas (Weinstein et al., 1998), bem como para que sejam elaboradas estratégias de prevenção de recaída, sendo útil que se ampliem pesquisas com este enfoque na área da dependência química (Schredl, 1999).

Kalra et al. (2000) realizaram uma pesquisa com pacientes psiquiátricos, entre eles dependentes químicos (28\%) que estavam utilizando psicofármacos como benzodiazepínicos, antidepressivos, antipsicóticos e estabilizadores de humor, durante o período de internação hospitalar, e analisou-se como eram os sonhos desta amostra $(n=50)$. Os sonhos desses pacientes foram comparados aos de voluntários normais, tendo sido obtidos os seguintes resultados: os pacientes recordaram menos os seus sonhos, seus conteúdos foram mais aterrorizantes e ocorreram mais repetições dos relatos, quando comparados com o grupo-controle. Essas diferenças ocorreram mesmo antes do aparecimento da doença mental. Após o início da farmacoterapia, $78 \%$ dos pacientes observados tiveram seus sonhos suprimidos, sendo que, do grupo que tomava benzodiazepínicos, apenas $18 \%$ não reduziu seus sonhos. Essa redução significativa associada aos benzodiazepínicos pode ser explicada pela sua ação diminuindo o sono REM e o estágio 4 do sono, fases estas relacionadas à produção do sonho. Kalra et al. (2000) referem, porém, que não é possível afirmar se os sonhos são suprimidos com o uso desse tipo de medicação ou se a sua ação causa apenas um prejuízo na memória onírica.

O álcool que, durante o seu uso, pode, a princípio, prolongar o sono e reduzir a quantidade de sono REM, quando é retirado, tem um efeito contrário: o sono é diminuído, a ansiedade aumenta e ocorrem muito mais sonhos pelo aumento compensatório do sono REM (Usher, 1991).

Drummond et al. (1998) pesquisaram alcoolistas na fase de desintoxicação e constataram que o sono desse grupo tem uma duração menor, é mais fragmentado e superficial no início da abstinência, melhorando lentamente no decorrer do primeiro ano, caso não ocorram recaídas.

Schredl (1999) investigou 74 pacientes 
alcoolistas no período de duas a quatro semanas após a retirada do álcool e constatou que a recordação dos sonhos por este grupo, na fase de desintoxicação, está levemente aumentada, o que poderia ser explicado por um aumento na freqüência de despertares noturnos. O despertar durante ou após a fase REM parece ocasionar o armazenamento do conteúdo sonhado na memória recente, o que facilitaria o seu resgate. A presença de pesadelos, é preciso salientar, foi comum nessa amostra.

Em sua pesquisa, Schredl (1999) observou que fatores como: dias de abstinência, duração do alcoolismo e a quantidade de álcool ingerida diariamente não foram relacionadas à freqüência da recordação dos sonhos, havendo, no entanto, uma correlação positiva com quantidade de sono REM, latência curta REM e uma elevada densidade do sono REM. Um estado negativo antes de dormir, nessa pesquisa, esteve associado a uma prevalência maior de emoções negativas no conteúdo dos sonhos.

Christo e Franey (1996) relatam que 84\% de 101 sujeitos pesquisados comentaram ter sonhado com substâncias psicoativas, o que ocorreu com maior freqüência quando estavam abstinentes. Sonhar com essas substâncias, nessa pesquisa, estava associado com a ocorrência de craving e insônia.

Há uma diferença significativa entre homens alcoolistas e não-alcoolistas quanto ao conteúdo de seus sonhos: os primeiros, ao contrário dos demais, costumam sonhar com a bebida alcoólica e com a transição para a sobriedade, também utilizando seus sonhos para expressar o craving, o que torna possível trabalhar a prevenção à recaída (Peters, 1997).

\section{Objetivo}

Estudar, em pacientes alcoolistas internados para desintoxicação, a associação entre a presença do conteúdo "álcool" nos sonhos e o aumento no craving, a gravidade da dependência, a quantidade de álcool consumida, a dose de benzodiazepínico utilizada e a fragmentação do sono.

\section{Método}

Este foi um estudo transversal, de associação entre variáveis, que utilizou uma amostra "por conveniência”. Fizeram parte da amostra 77 sujeitos dependentes do álcool, do sexo masculino, com grau mínimo de escolaridade de quinta série do ensino fundamental, internados em duas unidades especializadas em dependência química de Porto Alegre, Rio Grande do Sul - uma pública, a Unidade de Desintoxicação do Hospital Psiquiátrico São Pedro, e outra privada, a Clínica São José. O período de coleta dos dados foi entre outubro de 2001 e agosto de 2002.

A idade média apresentada foi de 41,5 anos $(\mathrm{SD}=8,8)$, sendo o limite inferior e o superior equivalentes a 18 e 63 anos, respectivamente. A última ingesta de bebida alcoólica devia ter ocorrido nas 24 horas precedentes à internação. Os sujeitos avaliados não podiam estar desorientados auto e alopsiquicamente, apresentar sintomas psicóticos ou depressivos, bem como ter déficit cognitivo ou sintomas de privação que alterassem a performance nos testes. Não foram incluídos os pacientes que apresentaram um quadro de delirium tremens durante a internação atual, bem como aqueles que dependiam de outras substâncias psicoativas, salvo a nicotina.

No total, foram excluídos 17 sujeitos, dos quais sete apresentavam sintomas psicóticos, dois tinham um quadro de delirium tremens e oito estavam abstinentes do álcool há mais de 24 horas, restando, então, os 77 participantes que compuseram esse estudo.

Foram utilizados como instrumentos: 1) Entrevista estruturada com o objetivo de definir o perfil sociodemográfico da amostra estudada, avaliar critérios de inclusão e exclusão e a quantidade de álcool consumida diariamente, sendo esta convertida em "Unidades Internacionais de Álcool" (Laranjeira e Pinsky, 1997); 2) Questionário Short-Form Alcohol Dependence Data - SADD criado por Raistrick (1983) e padronizado no Brasil por Jorge e Masur (1986) para avaliar o grau de gravidade da dependência do álcool; 3) Mini-Mental State Examination: MMSE (Folstein e McHugh, 1975) para excluir da amostra os pacientes com prejuízo cognitivo, sendo utilizado como ponto de corte o escore 25 ; 4) Questionário para avaliação do craving (Araujo, 2002) constituído por 11 questões objetivas que se referem ao craving avaliado nos três primeiros dias de desintoxicação. Esta escala classifica o craving em: insignificante (0-5 pontos), leve (6-14), médio (15-25) e forte (acima de 26 pontos); 5) Questionário para avaliar o sono e os sonhos (Araujo, 2002) composto por 19 questões objetivas que se referem ao sono e sonhos nos três primeiros dias de desintoxicação. As duas últimas escalas ainda não foram publicadas, mas encontram-se anexas à Dissertação de Mestrado de Araujo (2002). 
A presente pesquisa foi avaliada por um comitê de ética e, a partir de sua aprovação, foi iniciada a coleta de dados. Cada paciente que preencheu os critérios de inclusão foi avaliado no quarto dia de abstinência, individualmente, tendo sido realizada uma Entrevista estruturada e aplicados o Questionário SADD e o Mini-exame do Estado Mental.

Os pacientes que não preencheram nenhum critério de exclusão foram incluídos na amostra e, a estes, nesse momento, aplicou-se o questionário para mensurar o craving e o questionário do sono e dos sonhos.

Os testes estatísticos utilizados foram os descritivos para uma análise exploratória dos dados e o Teste Qui-Quadrado para verificar a existência de associação entre variáveis, tendo sido utilizado o nível de significância como parâmetro equivalente a 5\%.

\section{Resultados}

A média de idade com a qual os indivíduos experimentaram a bebida alcoólica foi equivalente a 18,9 anos $(\mathrm{SD}=6,7)$, sendo os limiares inferior e superior correspondentes a 8 e 40 anos. O número total de internações na vida de cada sujeito pesquisado teve uma média igual a 3,94 $(S \mathrm{SD}=5,3)$. A quantidade média de álcool consumida diariamente, antes da internação, foi de 31,7 UI (Unidades Internacionais); $\mathrm{SD}=22,6(\mathrm{n}=77)$. Com relação à gravidade da dependência, de acordo com a Escala SADD, 57,1\% dos sujeitos apresentaram um nível grave $(n=44), 32,5 \%$ moderado $(\mathrm{n}=25)$ e $10,4 \%(\mathrm{n}=8)$ leve. A média de pontos da amostra pelo Mini-Mental foi de 27,7 $(\mathrm{SD}=1,78)$, sendo o escore mínimo equivalente a 25 pontos (ponto de corte).

Quanto à análise descritiva do sono, observouse que $45,5 \%(n=35)$ dos sujeitos consideraram a fase inicial do sono como "muito boa", $22,1 \%(\mathrm{n}=17)$ "boa", 16,9\% ( $\mathrm{n}=13)$ "regular" e 15,6\% ( $=12)$ "muito ruim”. Quanto à fase intermediária, 22,1\% ( $\mathrm{n}=17)$ referiram nunca ter acordado, $27,3 \%(\mathrm{n}=21)$ acordaram em um dia, 23,4\% ( $\mathrm{n}=18)$ acordaram em dois dias e $27,3 \%(n=21)$ acordaram em três dias. A fase final do sono foi avaliada como "muito boa" para $64,9 \%(\mathrm{n}=50)$, como "boa" para $15,6 \%(\mathrm{n}=12)$, como "regular" para $10,4 \%(\mathrm{n}=8)$ e como "muito ruim" para $9,1 \%(\mathrm{n}=7)$.

Quanto à análise descritiva dos sonhos, 35,1\% $(\mathrm{n}=27)$ não sonharam em nenhuma noite, $32,5 \%$ $(\mathrm{n}=25)$ sonharam em "uma noite", $16,9 \%(\mathrm{n}=13)$ em "duas noites" e 15,6\% ( $\mathrm{n}=12)$ em "três noites".
Ao serem questionados quanto à ocorrência de pesadelos, $84,4 \%(\mathrm{n}=65)$ não apresentaram esse tipo de sonho, $13 \%(\mathrm{n}=10)$ apresentaram em "um dia", 1,3\% $(\mathrm{n}=1)$ em "dois dias", nenhum sujeito relatou ter tido pesadelos nos "três dias" e 1,3\% $(\mathrm{n}=1)$ "não lembrava”. O conteúdo "álcool” não apareceu nos sonhos de $72,7 \%$ dos indivíduos $(\mathrm{n}=56) ; 23,4 \%(\mathrm{n}=18)$ sonharam com álcool em "um dia", 3,9\% (n = 3) em "dois dias", e nenhum sonhou com álcool nos "três dias".

Foi utilizado o Teste Qui-Quadrado para analisar se havia uma associação entre apresentar despertares noturnos durante o sono e a produção de sonho, o que não pôde ser demonstrado $\left(\mathrm{x}^{2}=1,4154\right.$; $p=0,234)$. No entanto, ao se analisar essa característica do sono com o sonhar com o álcool, obteve-se uma associação significativa $\left(\mathrm{x}^{2}=17,492 ; \mathrm{p}<0,001\right)$ : os pacientes que apresentavam um aumento nos despertares noturnos relatavam mais este tipo de sonho.

Ao analisar a pontuação total da escala para avaliar o craving, obteve-se uma média igual a 12,2 pontos $(S D=10,5)$, tendo sido obtido nesta amostra o escore mínimo 0 (zero) e o máximo 40. Quando foi solicitado que dessem uma nota para intensidade média do craving durante a internação, obtiveram-se como resultados: $58,4 \%$ atribuíram nota zero $(n=45) ; 23,4 \%$ entre um e dois ( $\mathrm{n}=18) ; 7,8 \%$ entre três e quatro $(n=6) ; 2,6 \%$ entre cinco e seis $(n=2) ; 5,2 \%$ entre sete e oito $(n=4)$ e $2,6 \%$ entre nove e dez $(n=2)$.

A partir do cruzamento estatístico das variáveis "sonhar com álcool" e "craving", observou-se a existência de associação significativa entre ambas $\left(\mathrm{x}^{2}=14,281 ; \mathrm{p}<0,001\right)$. A média das notas da fissura do grupo que sonhou com álcool foi igual a 18,38 $(S D=10,45)$, enquanto a do grupo que não sonhou foi $8,08(S D=8,21)$.

Não pôde ser comprovada a associação entre sonhar com álcool e a dose utilizada de benzodiazepínico $\left(x^{2}=0,020 ; n . s\right)$, também não estando relacionada a dose desta medicação com o comportamento de sonhar, independentemente de seu conteúdo $\left(x^{2}=0,097 ; n . s\right)$.

Foi encontrada uma associação significativa entre o sonhar com o álcool e a gravidade da dependência avaliada pelo SADD (x2 = 7,298; $\mathrm{p}<0,01)$ e entre sonhar com o álcool e quantidade consumida dessa substância psicoativa ( $\mathrm{x} 2=9,032 ; \mathrm{p}<0,01)$ : aqueles alcoolistas considerados graves e que bebiam um número maior de unidades de álcool (UI) por dia apresentavam mais esse tipo de sonho. O comportamento de sonhar, independentemente de seu conteúdo, no entanto, não demonstrou estar associado à quantidade de álcool consumida ( $x^{2}=0,259 ;$ n.s. $)$. 


\section{Discussão dos resultados}

A relação entre "sonhar com álcool" e "craving" foi um dos resultados encontrados $\left(\mathrm{x}^{2}=14,281\right.$; $\mathrm{p}<0,001$ ), sendo que essa associação já havia sido descrita por alguns autores, não somente referindose ao álcool, como a outras substâncias psicoativas (Weinstein et al., 1998; Schredl, 1999; Christo e Franey, 1996; Peters, 1997; Colace, 2000).

Grande parte da amostra afirmou não ter sentido vontade de beber durante a internação: $58,4 \%$ atribuíram a nota zero à média do craving nesses dias, e a pontuação média, nesta escala específica, foi de 12,22 pontos - "fraca" ( $S D=10,55)$. Neste caso, o fato de os pacientes estarem internados em um ambiente protegido de estímulos envolvendo o álcool, pode-se inferir, atenuou o desejo de beber nessa amostra, o que estaria de acordo com o que foi escrito por Weinstein et al. (1998) e Edwards e Dare (1997), quando estes advertiram que as imagens mentais ligadas à bebida alcoólica e sugestões cognitivas internas ou ambientais interferem no aumento do craving, sendo importante a evitação destas para a manutenção da abstinência.

Cerca de $67,6 \%$ dos pacientes deste estudo, quando questionados a respeito de seu sono, relataram que a sua fase inicial, correspondente ao "pegar no sono", foi entre "muito bom" (45,5\%) e "bom" $(22,1 \%)$. Quanto ao final do sono - o período de despertar - 80,5\% consideraram sua qualidade entre "muito bom" $(64,9 \%)$ e "bom" $(15,6 \%)$, não tendo o sono sido interrompido cedo demais. Da amostra pesquisada, $22,1 \%$ nunca acordou durante a fase intermediária do sono, 35,1\% acordou no máximo uma vez, 14,3\%, no máximo duas, e 28,6\%, três ou mais vezes. Esta característica de fragmentação da fase intermediária do sono confere com o que foi descrito por vários autores que escreveram sobre o sono de alcoolistas na fase de desintoxicação (Schredl, 1999; Usher, 1991; Drummond et al., 1998).

Mesmo o sono apresentando-se de forma entrecortada para a maior parte da amostra, é preciso destacar que $80,5 \%$ julgaram que seu sono estava melhor nos três primeiros dias de abstinência se comparado com os dias em que estavam consumindo bebidas alcoólicas, contra apenas 5,2\% que o consideraram pior e $14,3 \%$ que não perceberam diferença entre ambos.

No período de privação do álcool há um aumento compensatório da fase REM (Usher, 1991), e a alta prevalência dos despertares durante essa fase do sono, nesses indivíduos, facilita que haja um resgate dos sonhos, já que seu conteúdo fica armazenado na memória recente (Schredl, 1999). Estes achados teóricos poderiam explicar o alto índice de pacientes que apresentaram algum sonho nessa amostra durante a internação: 64,9\%, sendo 32,5\% em "um dia"; $16,9 \%$ em "dois dias" e 15,6\% em "três dias".

Não houve uma associação significativa entre acordar durante a noite e uma quantidade maior de sonhos produzidos $\left(\mathrm{x}^{2}=1,415 ; \mathrm{p}=0,234\right)$, o que contradiz os resultados de Schredl (1999). Houve, por outro lado, uma associação entre esta característica do sono e sonhar com álcool ( $\left.\mathrm{x}^{2}=17,492 ; \mathrm{p}<0,001\right)$, o que se infere, com base no trabalho de Colace (2000), que o fato de sonhar com a substância psicoativa poderia estar relacionado à ansiedade e culpa, ocasionando o despertar.

Se o objeto da análise fosse o total da amostra, se poderia observar que não foi freqüente o relato de pesadelos (14,3\%), diferentemente do que foi encontrado por Shredl (1999), não sendo possível relacionar essas emoções negativas percebidas nos sonhos com os despertares noturnos.

O conteúdo "álcool” apareceu no relato dos sonhos dos indivíduos pesquisados, contudo, a freqüência com que isso ocorreu foi muito aquém do esperado: $72,7 \%$ não sonharam com álcool, 23,4\% sonharam com álcool em "um dia” e 3,9\%, em "dois dias". Este baixo índice contradiz o achado de Colace (2000) em dependentes de heroína, o de Christo e Franey (1996) em dependentes de múltiplas drogas, incluindo o álcool, e o de Peters (1997) e Johnson (2000) em alcoolistas.

É preciso destacar, no entanto, que essa pesquisa verifica os sonhos referentes apenas nos três primeiros dias de abstinência, sendo um tempo mais curto do que o delimitado nos demais estudos e, além disto, não faz uma comparação entre o grupo de alcoolistas e um controle, por meio do qual, provavelmente, esse comportamento relacionado ao conteúdo do sonho, nessa amostra específica, ficaria evidenciado.

A variável "sonhar com álcool”, no entanto, tem uma associação significativa com a gravidade da dependência mensurada pelo SADD $\left(x^{2}=7,298 ; p<0,01\right)$ e com a quantidade de álcool consumida $\left(x^{2}=9,032\right.$; $\mathrm{p}<0,01$ ): os pacientes alcoolistas "graves" e que consumiam maior quantidade de bebida alcoólica apresentavam mais esse tipo de sonho.

A quantidade de álcool consumida não estava associada a um aumento na produção de sonhos de forma geral $\left(x^{2}=0,259 ; n . s\right)$, o que estaria de acordo com os achados de Schredl (1999) e que poderia ser 
explicado por um mecanismo neuroquímico (Usher, 1991), mas estava associada, sim, especificamente, a um aumento do número de sonhos com álcool, o que demonstra o envolvimento de mecanismos psicológicos nesse processo.

Como o uso de benzodiazepínicos na síndrome de privação do álcool é uma conduta preconizada no alívio da sintomatologia (Ramos e Galperim, 1997) e como essa medicação tem um efeito depressor do sistema nervoso central, interferindo de forma a reduzir o sono REM e, em decorrência, a produção de sonhos (Kalra et al., 2000), foi analisada a associação entre a dosagem desse fármaco e o comportamento de sonhar, com ou sem o conteúdo "álcool".

Não foi encontrada, como foi no trabalho de Kalra et al. (2000), uma associação entre a dose de benzodiazepínico utilizada por dia e a produção de sonhos $\left(x^{2}=0,097 ; p=0,754\right)$, bem como uma associação dessa medicação com o sonhar com o álcool (x2 = 0,02; $\mathrm{p}=0,908)$, o que poderia ser uma variável interveniente na análise dos resultados.

Na medida em que se constata haver uma associação entre sonhar com o álcool e um aumento no craving e se reconhece a participação da vontade de consumir a bebida alcoólica como sendo uma freqüente causa de recaída (Beck et al., 1999; Hogstrom et al., 1999; Peters, 1997; Marlatt e Gordon, 1993), pode-se perceber a importância de os sonhos serem mais bem aproveitados pelos profissionais que atendem a dependentes químicos.

\section{Conclusões}

Uma das grandes surpresas deste estudo foi verificar que pacientes alcoolistas internados para desintoxicação, impedidos de beber não só por questões referentes à vontade própria, mas por contingências ambientais que o impossibilitam de ter acesso ao álcool, não apresentam uma intensa vontade de beber.

É interessante pensar neste ponto e talvez dar-se conta a respeito da idéia de que a internação hospitalar para desintoxicação deve oferecer, de alguma forma, um local de "continência" para esse desejo tão difícil de ser aplacado como é o de beber ou de consumir drogas e que, muitas vezes, não consegue ser controlado em regime ambulatorial.

Não se pode deixar de informar que o fato de alguns pacientes quererem demonstrar uma "melhora" em seu quadro para, conseqüentemente, antecipar a sua alta seja um viés quando se analisa a variável craving durante uma internação hospitalar.
Também se imaginava que sonhar com o álcool seria um comportamento freqüente nesses indivíduos que até a véspera da internação consumiam bebida alcoólica diariamente, ou quase nessa medida.

Foi interessante constatar que os pacientes avaliaram seu sono na fase de desintoxicação como sendo de melhor qualidade do que o apresentado durante o consumo e que, devido a este ter interrupções em sua fase intermediária, favoreceu para que os sonhos fossem recordados.

O tema craving é comum de ser escutado na clínica do dependente químico e pode aparecer a qualquer momento, mesmo após um longo tempo em abstinência. Caso vença a luta contra o controle do indivíduo, poderá ser o desencadeador de uma recaída.

O problema é que, em grande parte das ocasiões, ele aparece sem aviso prévio, ou que os terapeutas, juntamente com os pacientes, não conseguem detectálo a tempo, dificultando o seu manejo e diminuindo a auto-eficácia do sujeito que, com menos estratégias de enfrentamento, termina por sucumbir ao álcool.

Por isso, saber da associação entre sonhar com álcool e o craving nos fornece uma arma importante para detectarmos a presença dessa "vontade" antes que ela venha acompanhada de maiores prejuízos.

No caso de dependentes químicos, sabe-se que os sonhos ainda são pouco utilizados na clínica, principalmente entre os profissionais que trabalham com a Terapia Cognitivo-Comportamental.

Esta pesquisa não tem, portanto, a pretensão de esgotar a discussão dos resultados encontrados, mas sim aprofundar o tema "sonhar com álcool e craving", de modo a suscitar o interesse para que novas investigações sejam feitas, tendo por foco os sonhos e sua utilização na área da dependência química. 


\section{Referências bibliográficas}

Araujo, R.B. - A relação entre sonhos e craving em alcoolistas na fase de desintoxicação. Dissertação de Mestrado, Faculdade de Psicologia da Pontifícia Universidade Católica do Rio Grande do Sul, Porto Alegre, 2002.

Beck, A.T.; Wright, F.D.; Newman, C.F.; Liese B.S. - Terapia Cognitiva de las Drogodependencias, Paidós, Barcelona, 1999.

Christo, G.; Franey, C. - Addicts’ Drug-Related Dreams: Their Frequency and Relationship to Six-Month Outcomes. Substance Use \& Misuse 31(1): 1-15, 1996.

Colace, C. - Dreams in Abstinent Heroin Addicts: Four Cases Reports. Sleep and Hypnosis 2 (4): 160-3, 2000.

Drummond, S.P.; Gillin, J.C.; Smith, T.L.; Demodena, A. - The Sleep of Abstinent Pure Primary Alcoholic Patients: Natural Course and Relationship to Relapse. Alcoholism: Clinical and Experimental Research 22 (8): 1796-802, 1998.

Edwards, G.; Dare, C. - Psicoterapia e tratamento das adições. Artes Médicas, Porto Alegre, 1997.

Hogstrom, B.A.; Thorburn, D.; Hiltunen, A.; Borg, S. - Prediction of Single Episodes of Drinking during the Treatment of Alcohol-Dependent Patients. Sweden: Alcohol 18 (1): 35-42, 1999.

Folstein, M.; Mchugh, P.R. - Mini-mental state: A Practical Method for Grading the Cognitive State of Patient for the Clinician. Journal of Psychiatry Research 12: 189, 1975

Johnson, R.A. - Relationship of Dreams of Drinking and Negative Expectancies to Alcohol Treatment Outcome in a Veterans Administration Alcoholic In-patient Treatment Population. Dissertation. Abstracts. International; Section B, The Sciences and Engineering 61 (6-B): 328, 2000
JoRGE, M.R.; MANSUR, J. - Questionários padronizados para avaliação do grau de severidade da Síndrome de Dependência do Álcool. Jornal Brasileiro de Psiquiatria 35 (5): 287-92, 1986.

Kalra, R.; Natu, M.V.; Deswal, R.; Agarwal, A.K. - Dream Features.in Psychiatric Patients on Multiple Psychoactive Drugs. Human Psychopharmacology Clinical Experimental 15: 525-8, 2000.

Laranjeira, R.; Pinsky, I. - Alcoolismo (Coleção Mitos e Verdades). Contexto, São Paulo, 1997.

Marlatt, A.; Gordon, J. - Prevenção de Recaída - Estratégias de Manutenção no Tratamento de Comportamentos Adictivos. Artes Médicas, Porto Alegre, 1993.

Peters, K.A. - The Dreams of Alcoholic Men in Early Sobriety. Dissertation Abstracts International, Section B, The Sciences and Engineering 57 (10-B): 6588, 1997.

RAISTRICK, D. ET AL. - Development of a Questionnaire to Measure Alcohol Dependence. British Journal of Addiction 78: 89-95, 1983.

Ramos, S.; GAlPERIM, B. - Desintoxicação. In Ramos, Sérgio; Bortolote, J.M. Alcoolismo Hoje: 149-59. Artes Médicas, Porto Alegre, 1997.

Reimão, R. - Sono - Estudo Abrangente. Atheneu, São Paulo, 1996

Schredl, M. - Dream Recall in Patients With Primary Alcoholism after Acute Withdrawal. Sleep and Hypnosis 1 (1): 35-40, 1999.

Usher, R. - O sono. Saraiva, São Paulo, 1991.

Weinstein, A.; Lingford, H.A.; Martinez, R.J.; Marshall, J. - What makes Alcohol-dependent Individuals Early in Abstinence Crave for Alcohol: Exposure to the Drink, Images of Drinking or Remembrance of Drinks Past? Bristol: Alcoholism: Clinical and Experimental Research 22 (6): 1376-81, 1998. 